\title{
EFFECT ON A SHOCK WAVE BOUNDARY LAYER INTERACTION OF AIR JET VORTEX GENERATORS
}

\author{
L. J. Souverein ${ }^{1,2}$ and J.-F. Debiève ${ }^{1}$ \\ ${ }^{1}$ Institut Universitaire des Systèmes Thermiques Industriels (IUSTI) \\ Supersonic Group \\ Rue Enrico Fermi 5, 13453 Marseille Cedex 13, France \\ ${ }^{2}$ Delft University of Technology \\ Faculty of Aerospace Engineering \\ Kluyverweg 1, 2629 HS Delft, The Netherlands
}

The effect of upstream injection by means of continuous Air Jet Vortex Generators (AJVGs) on a shock wave turbulent boundary layer interaction is experimentally investigated. The baseline interaction is of the impinging type, with a flow deflection angle of $9.5^{\circ}$, a Mach number $\mathrm{M}_{e}=2.3$, and a momentum thickness based Reynolds number of 5,000. Considered are the effects of the AJVGs on the upstream boundary layer flow topology and on the spatial and dynamical characteristics of the interaction. To this aim, Stereoscopic Particle Image Velocimetry has been employed, in addition to hot-wire anemometry (HWA) for the investigation of the dynamical characteristics of the reflected shock. It is shown that the AJVGs significantly modify the three-dimensionality of the upstream boundary layer. Overall, the AJVGs cause a reduction of the separation bubble length and height. In addition, the energetic frequency range of the reflected shock is increased by approximately $50 \%$, which is in qualitative agreement with the smaller separation bubble size.

\section{INTRODUCTION}

The effect of a planar shock impinging on a turbulent boundary layer establishes one of the classic interaction phenomena in compressible viscous flow analysis. This particular form of interaction also has a direct technological relevance to

This is an Open Access article distributed under the terms of the Creative Commons Attribution-Noncommercial License 3.0, which permits unrestricted use, distribution, and reproduction in any noncommercial medium, provided the original work is properly cited. 
the performance of high-speed vehicles, affecting notably, for example, the efficiency of supersonic intakes. Furthermore, maximum mean and fluctuating pressure and thermal loads on a structure are most often found in regions of shock wave boundary layer interaction (SWBLI) and are, thus, important factors in vehicle development. Flow control is seen as an important issue in future vehicle design [1] to negate these adverse effect. In this context, the European 6th framework program UFAST "Unsteady effects in shock wave induced separation" was recently initiated.

In the case of a shock wave turbulent boundary layer interaction, provided a sufficiently strong adverse pressure gradient, the boundary layer separation can occur. The resulting bubble of reversed flow has been observed to pulse, leading to low frequency oscillations of the reflected shock, cause of the detrimental unsteady fluctuations. One of the open issues is the source of the pulsation [2-4]. Two principal mechanisms have been proposed, ascribing the unsteadiness either to large-scale elongated structures in the upstream boundary layer, or to a mechanism based on the entrainment of mass by the shedding of large-scale coherent structures. Various systems have been imagined to reduce the separation bubble with the aim of suppressing the unsteadiness. The most sophisticated concern the dynamic control with a feedback loop. A more crude solution consists of static systems based on the generation of vortices near the wall upstream of the interaction. One can think of subvortex generators that consist of mechanical systems fixed at the wall. Another example is based on the injection of fluid by means of continuous AJVGs in upstream boundary layer. This approach is of particular interest due to the potential of integrating flow control with transpiration cooling. Since this case has been studied principally in the transonic or low supersonic flows, the aim of the current work is to examine this problem in the fully supersonic flow domain where only limited data are available.

In the following, the effect of upstream injection by means of continuous AJVGs on a shock wave turbulent boundary layer interaction is experimentally investigated in the case of a supersonic flow with a Mach number of $\mathrm{M}_{e}$ $=2.3$. The associated flow deflection angle is $9.5^{\circ}$, leading to a significant mean separation bubble.

\section{EXPERIMENTAL ARRANGEMENT AND FLOW CONDITIONS}

The experiments have been performed in the S8 Supersonic wind tunnel at the IUSTI in Marseille. The tunnel has been equipped with a special measurement section to perform the experiments with air jet vortex generator control. The flow conditions and the setup of the control experiment are detailed in the following. 


\subsection{Flow Facility}

The wind tunnel is a closed-loop continuously running with a particularly low free-stream turbulence, the Mach number is 2.3, the unit Reynolds number of $5.5 \cdot 10^{6} \mathrm{~m}^{-1}$, the stagnation temperature $T_{0}=295 \mathrm{~K}$, and a total pressure of $p_{0}=0.5 \mathrm{~atm}$. At the inlet conditions, for the interaction correspond a Reynolds number based on momentum thickness of approximately $\operatorname{Re}_{\theta}=5,000$, a friction coefficient of $C_{f}=2.1 \cdot 10^{-3}$, and a boundary layer thickness of $\delta_{0}=10 \mathrm{~mm}$. An extensive description of the flow facility can be found in [5].

\subsection{Control Experiment Setup}

A row of AJVGs was placed upstream of the interaction to study the effect of upstream disturbances on the mean and unsteady flow characteristics for the control of a SWBLI. The vortex generators consist of a row of ten holes, with a spanwise pitch of about one boundary layer thickness. The diameter of the holes is $\phi=0.8 \mathrm{~mm}(\phi<\delta / 10)$. The row is perpendicular to the flow. The axis of the holes is inclined within the spanwise-wall-normal-plane under an angle of $\psi=45^{\circ}$. The AJVG array has been located at around $5 \delta$ upstream of the zone of reflected shock oscillations. A settling chamber is installed underneath the complete array of AJVGs to assure a homogeneous and stable air injection. The temperature in the chamber is around the stagnation temperature of the channel flow. It was verified that the pressure spectrum in the chamber filled with the porous medium does not present any resonant peaks. The stagnation pressure in the chamber is chosen at $P_{0 \text { jets }}=0.4$ bar, close to the stagnation pressure of the tunnel $\left(p_{0}=0.5 \mathrm{~atm}\right)$.

The injected airflow was found to be negligible as compared to the mass flow deficit of the boundary layer: for an injection pressure of $P_{0 \text { jets }}=0.4$ bar and considering the row of 10 injectors over a span of $\Delta Z=100 \mathrm{~mm}$, given the boundary displacement thickness of $\delta^{*}=3 \mathrm{~mm}$, the ratio of the jets mass flow to the boundary mass flow deficit is

$$
\frac{\text { Jet flux }}{\rho_{e} U_{e} \delta^{*} \Delta Z} \approx 3 \% \text {. }
$$

\subsection{Flow Diagnostics Methods}

For the HWA, the constant temperature system 'Streamline Dantec CTA' was used in the balanced bridge mode. The diameter of the hot wire was $5 \mu \mathrm{m}$ and the overheat ratio was 0.6. The data were sampling with a National Instrument recorder NI6133 using approximately $2 \cdot 10^{6}$ samples. 
The Particle Image Velocimetry (PIV) investigation was made using a Dantec Dynamics system and software. The light sheets were generated by a double pulse ND:YAG laser New wave Solo II, which delivered $30 \mathrm{~mJ}$ per pulse, with a pulse delay set in the range of $1-2 \mu \mathrm{s}$. The light sheet thickness was $1 \mathrm{~mm}$. Incense smoke was used to seed the boundary layer. The particles were injected from the wall, upstream of the wind tunnel nozzle. The time constant of the particles was estimated to be $4.5 \mu \mathrm{s}$, corresponding to a diameter of $0.5 \mu \mathrm{m}[6]$. The particle images were recorded by Flowsense 10-bit cameras with a CCD (charge-coupled device) size of $1600 \times 1200$ pixels, equipped with Nikon Macro Nikkor $f=60 \mathrm{~mm} f / 2.8$ objectives with the diaphragm set to $f_{\#}=2.8$. The acquisitions were made using Flowmanager 4.71 software via the Dantec Flowmap System Hub. A peculiarity of this system is an internal storage, and therefore, long data acquisitions at high rate are possible $(12 \mathrm{~Hz}$ using the two cameras in half frame mode). The maximum of 10,000 image pairs were acquired with two cameras (5000 per camera). The images were processed with DynamicStudio2.00, statistics and postprocessing were done with in-house Matlab routines.

\subsection{General Description of the Flow}

The flow topology is depicted in Fig. 1, showing a Schlieren visualization of the interaction with and without control. As can be observed, the fully turbulent boundary layer which develops on the tunnel floor is subjected to a shock wave produced by a full-span sharp edge plate placed in the external flow. The imposed flow deflection angle is $9.5^{\circ}$, corresponding to a well developed separation. The baseline interaction has been extensively documented in literature $[5,7,8]$. As can be observed, the boundary layer is first perturbed by the AJVG array, which is located at the source of the weak shock-expansion system located upstream of the interaction. More downstream, the incident shock wave impacts on the boundary layer, causing the boundary layer to thicken and to separate. The jets cause a thickening of the reflected shock, indicative of either an increased

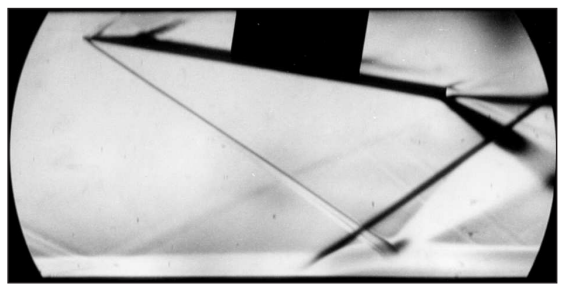

(a)

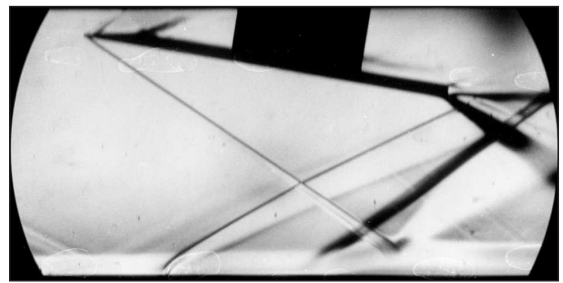

(b)

Figure 1 Schlieren visualisation of the interaction: (a) baseline interaction without AJVGs; and (b) modified interaction with AJVGs 


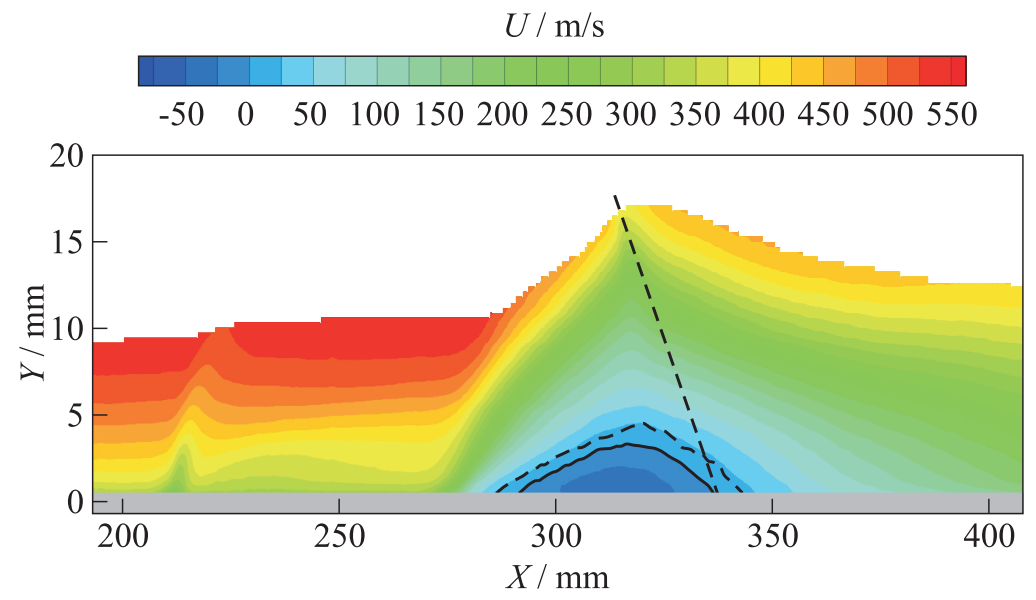

Figure 2 Mean longitudinal velocity component in the streamwise-wall normal plane with the minimum interaction length $(Z=-2.5 \mathrm{~mm}$ ), AJVGs on. (Refer Souverein and Debiève, p. 145.)

unsteadiness (shock excursion amplitude) or an increase in three-dimensionality (due to spanwise rippling). As can be observed, the interaction length (distance at the wall between the extrapolated incident and reflected shock) is not significantly affected.

The associated mean streamwise velocity is presented in Fig. 2. The flow is from left to right, showing the undisturbed incoming boundary layer on the left-hand side of the domain of interest. As can be seen, the boundary layer is perturbed by the jet array at $X=212.5 \mathrm{~mm}$. The boundary layer thickens, but without a change is free-stream velocity, indicating that the aforementioned shock-expansion system is of weak strength. The reflected shock foot is located at approximately $X=270 \mathrm{~mm}$, where the flow is lifted away from the wall and a separation bubble appears. The solid black contour line indicates the contour of zero velocity. The dashed contour represents the extent of the zero velocity contour for the undisturbed case. The dashed line indicates the extrapolated incident shock, impacting at $X=337 \mathrm{~mm}$. As can be observed, the jets significantly decrease the separation bubble size. In the following sections, the effect of the jets will be quantified in more detail.

\section{MODIFICATION OF THE UPSTREAM BOUNDARY LAYER BY AIR INJECTION}

To visualize the effect of the jets on the incoming boundary layer topology, threecomponent PIV-measurements have been made in the horizontal plane at four 


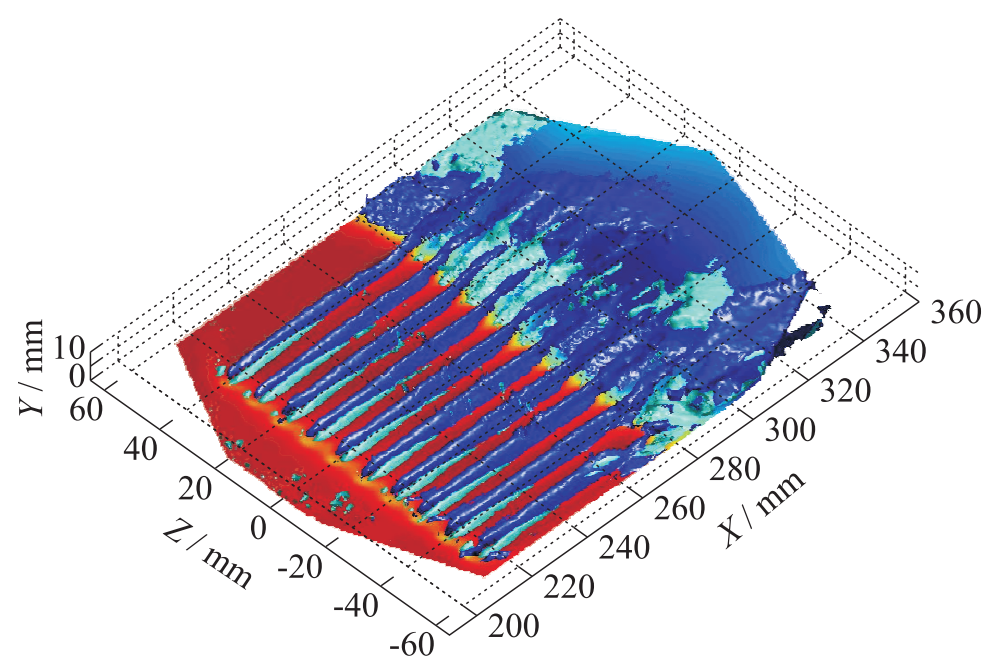

Figure 3 Isosurfaces of the angular velocity (blue: $\alpha=-5 \cdot 10^{3} \mathrm{rad} / \mathrm{s}$ and cyan: $\left.\alpha=5 \cdot 10^{3} \mathrm{rad} / \mathrm{s}\right)$, superimposed on contour of the mean longitudinal velocity at $Y=1 \mathrm{~mm}$. (Refer Souverein and Debiève, p. 146.)

heights, allowing the reconstruction of the mean three-dimensional (3D) flow field encompassing the complete domain of interest from the incoming boundary layer up to reattachment. For this data volume, the angular velocity around the local velocity vector has been computed. Figure 3 shows the resulting isosurfaces for values of $-5 \cdot 10^{3} \mathrm{rad} / \mathrm{s}$ and $5 \cdot 10^{3} \mathrm{rad} / \mathrm{s}$ superimposed on a contour map of the streamwise mean velocity component at $Y=1 \mathrm{~mm}$.

As a first observation, it is noted that the jets induce a spanwise asymmetry, skewing the flow with a small angle of approximately $2.8^{\circ}$ with respect to the tunnel axis. Second, the flow is modulated in the spanwise direction. Pairs of counterrotating longitudinal vortices, which are induced by each jets, are at the origin of this spanwise modulation. The angular velocity shows (in blue) the main vortices produced by the AJVGs, having negative angular velocity values (turning counterclockwise (ccw) when looking downstream along the coordinate axis). Also visible are (in cyan) small secondary vortex tubes with a positive angular velocity, which turn clockwise (cw) when looking downstream. These correspond to small secondary vortices generated between the jets and the wall, below the jets (the main vortices are generated between the jets and the outer flow, above the jets).

A zoom of the topology of these vortex is presented in Fig. 4. As can be observed from this figure, the mean velocity in-between the jets is increased from $U=350$ to $380 \mathrm{~m} / \mathrm{s}$. Since the velocity increase is directly associated to the two vortices, it seems to be an induced effect of the rotation of the longitudinal vortex 


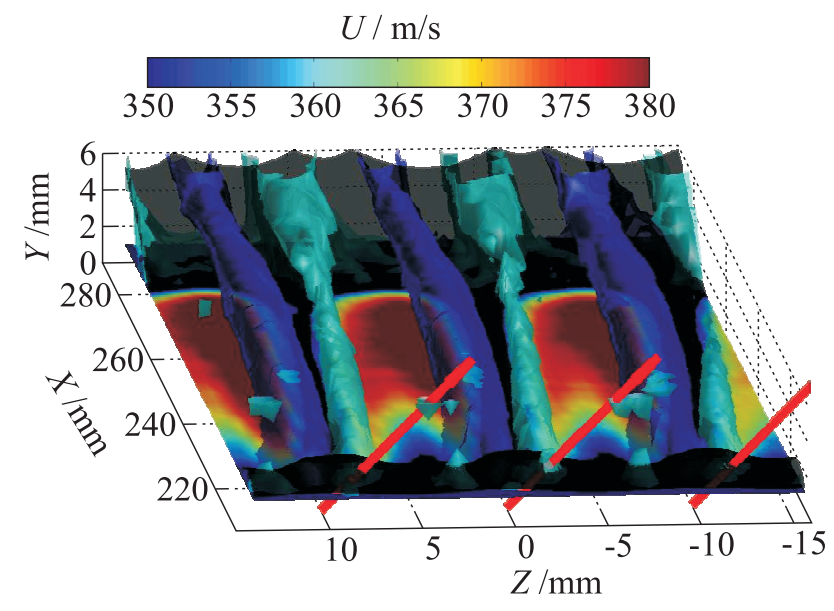

Figure 4 Isosurfaces of angular velocity, jets (blue: $\alpha=-5 \cdot 10^{3} \mathrm{rad} / \mathrm{s}$ and cyan: $\left.\alpha=1 \cdot 10^{3} \mathrm{rad} / \mathrm{s}\right)$. Black isosurface represents longitudinal velocity isocontours of $U=350 \mathrm{~m} / \mathrm{s}$ (low-speed fluid). Contours indicate longitudinal velocities at $Y=1 \mathrm{~mm}$, as indicated by the colour bar. Red arrows represent the jet location and injection direction. (Refer Souverein and Debiève, p. 147.)
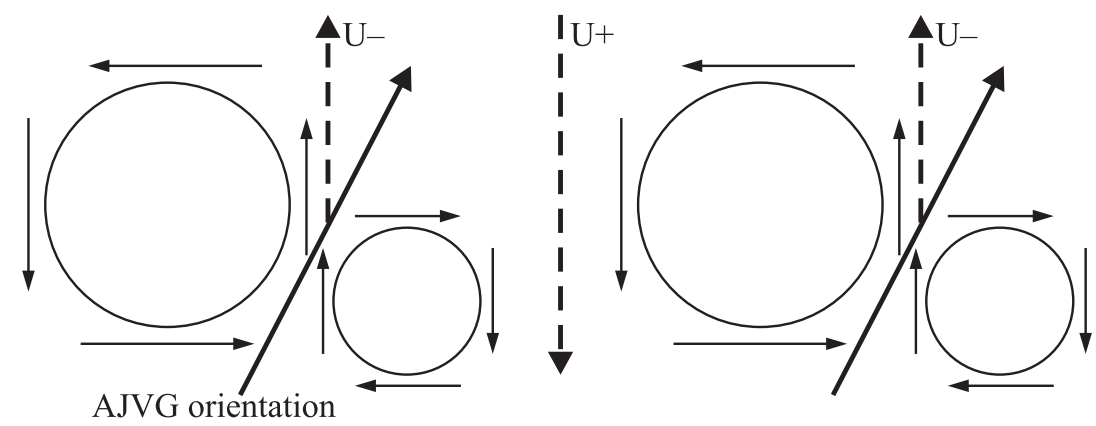

Figure 5 Schematic representation of the longitudinal vortices generated by the AJVGs, viewed in upstream direction. The bold arrow represents the jet

pairs, which transport fluid from higher up in the boundary layer towards the wall. At the same time, the mean velocity behind each jet is reduced, most likely as a result of the transport of low-speed fluid away from the wall by the vortices, in combination with the generation of a wake by the jets themselves.

From the preceding plots, the following vortex structure can be intuited, as illustrated schematically in Fig. 5 (looking in upstream direction with the negative spanwise coordinate pointing left). An estimate of the angular velocity 
for the large ccw vortex at mid-distance between jets and shock-foot can be obtained as follows:

$$
\begin{array}{ll}
\text { diameter: } & D=6 \mathrm{~mm} ; \\
\text { mean out of plane velocity: } & W=10 \mathrm{~m} / \mathrm{s} ; \\
\text { horizontal velocity component: } & V_{1}=30 \mathrm{~m} / \mathrm{s}(\text { for } Y=1 \mathrm{~mm}), \\
& V_{4}=10 \mathrm{~m} / \mathrm{s}(\text { for } Y=4 \mathrm{~mm}) ; \\
\text { distance from jet to interaction: } & L=50 \mathrm{~mm} ; \\
\text { longitudinal velocity: } & U_{1}=350 \mathrm{~m} / \mathrm{s}(\text { for } Y=1 \mathrm{~mm}), \\
& U_{4}=430 \mathrm{~m} / \mathrm{s}(\text { for } Y=4 \mathrm{~mm}) ; \\
\text { rotation rate: } & \alpha=\left(V_{4}-V_{1}\right) /(2 \pi D)=40 /\left(2 \pi 6 \cdot 10^{-3}\right) \\
& =1000 \mathrm{~Hz}=6.7 \cdot 10^{3} \mathrm{rad} / \mathrm{s} .
\end{array}
$$

This value is in good agreement with the values for the isocontours in Fig. 4. The travel time from jets to interaction is given by:

$$
\tau=\frac{2 L}{U_{4}+U_{1}}=\frac{2 \cdot 50 \cdot 10^{-3}}{350+430}=128 \mu \mathrm{s},
$$

the following number of rotations executed by the large ccw from its generation until the interaction is obtained:

$$
\alpha \tau=0.13
$$

Performing the same estimation just behind the jet, where the out of plane velocities are stronger, leads to a value of $\alpha \tau=0.31$. So, the total number of rotations may be expected to be around $1 / 4$, certainly, less than 1 . This means that the mixing induced by the rotation of the longitudinal vortices is limited.

The obtained mean longitudinal velocity profiles at $X=260 \mathrm{~mm}$, just upstream of the reflected shock foot, are visualized in Fig. 6 . Shown are the profile for the reference case without jets $\left(L_{\text {ref }}, 1\right)$ and two profiles with jets $(2$ and 3$)$. In accordance with the spanwise modulation of the flow, the two profiles with jets represent the two extremes of the AJVG effectiveness: $L_{\min }$ corresponds to the fullest profile, leading to the smallest local separation length, and $L_{\max }$ represents the profile with the largest velocity deficit, inducing the largest local separation length for the case with jets. This significant deficit does not lead to a local anticipated separation with respect to the reference case, however. It might, therefore, be that the governing parameter for the separation location is the flow very near the wall (below $y / \delta<0.1$ ). It is remarked that all profiles are self-similar in the outer part of the boundary layer $(y / \delta>0.8)$. It is noted that the jets may cause a slight increase in boundary layer height, but the increase falls within the measurement error. The increase in velocity observed in Fig. 4 corresponds well to the increase in fullness of the boundary profile for $L_{\min }$. 


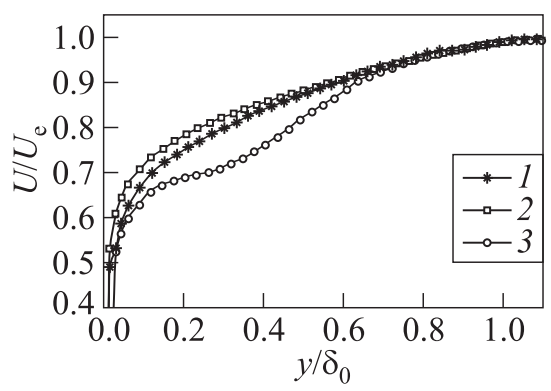

Figure 6 Longitudinal velocity profiles in the incoming boundary layer at $X$ $=260 \mathrm{~mm}: 1$ - reference profile without jets; and profiles with AJVGs control $(2-$ minimal separation and 3 - maximal separation), jet 0.4 bar

Using the rotation rate above and an estimated radius of $3 \mathrm{~mm}$ for the ccw vortex, it is estimated that the induced vertical displacement caused by the vortex rotation is $2 \mathrm{~mm}(y / \delta=0.2)$. Considering the reference boundary layer profile, such a displacement can, indeed, be held responsible for the change in fullness of the profiles with AJVGs and, hence, the modulation of the mean longitudinal velocity at $Y=1 \mathrm{~mm}$ observed in Fig. 4 . This seems to confirm the mechanism proposed in Fig. 5.

\section{MODIFICATION OF SEPARATION}

The effect of injection on the mean flow topology has been investigated. The mean streamwise velocity component in the wall parallel plane at $Y=1 \mathrm{~mm}$ is shown in Fig. 7 for the case with or without control jets. The solid black contour lines in this figure indicate the streamwise velocity for $200 \mathrm{~m} / \mathrm{s}$ (taken as indicative for the extrapolated reflected shock foot location) and for $0 \mathrm{~m} / \mathrm{s}$ (representing the detachment line and the reattachment line and, hence, the extent of the separation bubble). The dashed lines indicate for reference the respective contours for the case without jets. It was found that the $200 \mathrm{~m} / \mathrm{s}$ velocity contour at the reflected shock foot location becomes rippled by the jets, but that its mean spanwise position is only mildly affected, being pushed only slightly downstream as compared to the baseline interaction. This is in accordance with the thickening of the reflected shock observed in Fig. 1.

Considering the separation bubble, it is clear that the separation line becomes highly corrugated as well in the injection case. This effect is more pronounced than the corrugation of the reflected shock. The reattachment line is displaced upstream with respect to the undisturbed case, but it shows no signs of corru- 


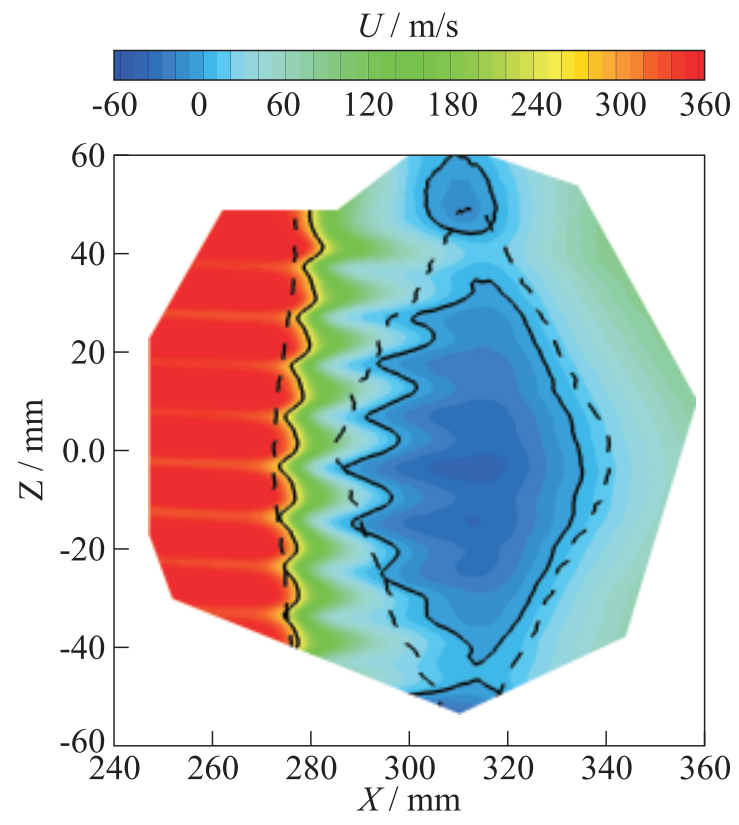

Figure 7 Mean longitudinal velocity component with AJVG control, $Y=1 \mathrm{~mm}$. (Refer Souverein and Debiève, p. 150.)

gation. Hence, the effect of the jets is to decrease the separation length at each spanwise location.

As a general remark, it is observed that although clear traces of AJVG induced longitudinal vortices exist upstream of the separation bubble, no trace of such vortices is found downstream of the interaction: the reattachment line is uncorrugated, and no sign of the vortex-patterns is visible downstream of the reattachment. So, either the longitudinal vortices are lifted over the interaction by the separation bubble and do not reappear at a height of $1 \mathrm{~mm}$, or they are destroyed by the unsteady processes occurring in the interaction region.

As was shown in the previous section, the AJVGs appear to induce longitudinal vortices that entrain high-speed fluid from higher up in the boundary layer. This fluid slightly displaces the reflected shock foot downstream and reduces the separation length. The effect on the separation line is more pronounced than the effect on the reflected shock. To quantify this effect, Fig. 8 shows the velocity distribution at $Y=1 \mathrm{~mm}$ for $L_{\mathrm{ref}}, L_{\mathrm{min}}$, and $L_{\max }$.

As can be observed, the reattachment point with AJVGs is moved upstream as compared to the reference case. Furthermore, the separation point is moved downstream for $L_{\min }$, while it is identical for $L_{\max }$ and $L_{\text {ref }}$. Hence, the sepa- 


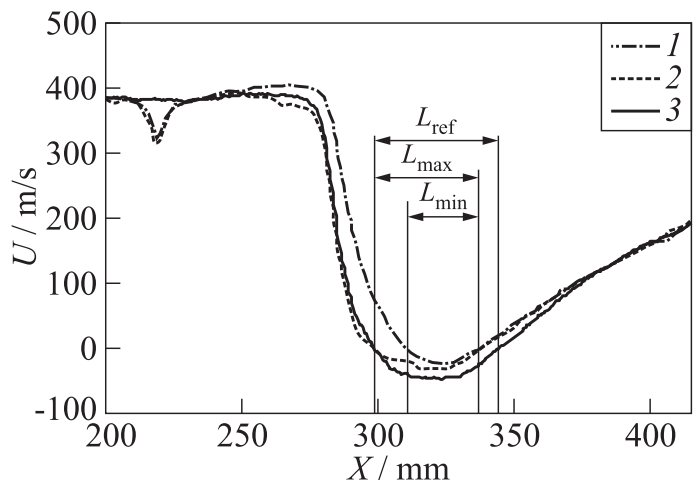

Figure 8 Longitudinal mean velocity profiles at $Y=1 \mathrm{~mm}$; the vertical lines indicate the separation point and the reattachment point for the cases with $\left(1-L_{\min }\right.$ and $2-$ $L_{\text {max }}$, jet 0.4 bar) and without (3) AJVG control

ration length for $L_{\text {min }}$ is significantly smaller than for $L_{\text {ref }}$, while $L_{\max }$ is only slightly smaller than $L_{\text {ref }}$. Downstream of the interaction, all cases attain the same mean velocity, and the effect of the jets, hence, disappears completely. The dip in the velocity in the upstream boundary layer indicates the location of the jets. As can be seen, the velocity for $L_{\min }$ increases slightly between the jets and the separation region, while the velocity for $L_{\max }$ decreases. This is due to the slight skewing of the flow by the action of the jets, as observed previously, while the velocity distributions have been obtained in planes parallel to the tunnel axis. In addition to reducing the separation length, the AJVGs also reduce the separation bubble height, as has been observed in Fig. 2, with the largest reduction corresponding to the smallest separation length. The jets cause an overall decrease in separation length and an accompanying decrease in maximum reverse flow velocity.

Concerning the modulation of the boundary layer profile, it has been found that a decrease in friction coefficient for the jet 'wake' $\left(L_{\max }\right)$ is linked to a larger separation length. On the contrary, an increase of both quantities for the fullest profile in-between the longitudinal vortices induced by the jets leads to a smaller separation length $\left(L_{\min }\right)$. The inverse effect has been observed for the shape factor, which is the largest for the slim profile and the smallest for the full profile. It seems, therefore, that the most important effect of the AJVGs is to modify the integral boundary layer parameters. There is a direct link between a reduction in separation length and the shape factor and the friction coefficient. This link is not obvious when comparing the reference profile without jets to the two profiles with jets. This might be due to 3D effects. Overall, the action of the jets is to reduce the bubble size. 


\section{MODIFICATION OF THE SHOCK DYNAMICS}

The intermittency in the shock position had been detected in the free-stream using hot wire. The root-mean square (RMS) values of the HWA-signal induced by the passage of the shock are presented in Fig. $9 a$ for different longitudinal positions in the symmetry plane of the wind tunnel with and without AJVG control. The maximum value of the RMS can be associated with the median shock location. A downstream shift in this location can be observed for the AJVG control case. This confirms the fact that the interaction length is slightly reduced with AJVG control. However, the shock excursion amplitude $L_{\text {ex }}$ (indicated by the width of the peak) is not significantly altered. The observed thickening of the shock in Fig. 1 cannot, therefore, be attributed to an increased shock excursion length.

The shock frequency range had been detected in the free stream using a hot wire positioned on the median position of the separation shock. The resulting spectra of the HWA signal for the cases with and without injection is shown in Fig. $9 b$. The spectrum is shown in premultiplied form $(f E(f)$ vs. $\log (f)$ where $f$ is the frequency) to correctly represent the energy concentration. The zone of maximum of spectral energy is not well defined but one can notice a significant shift in the energy bump of the spectrum to higher frequencies when the jets are activated. This is in agreement with a quasi-constant Strouhal number for the shock frequency:

$$
\mathrm{St}=\frac{f h}{U}
$$

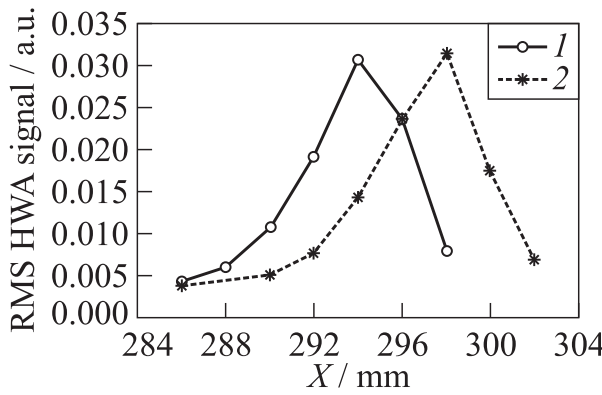

(a)

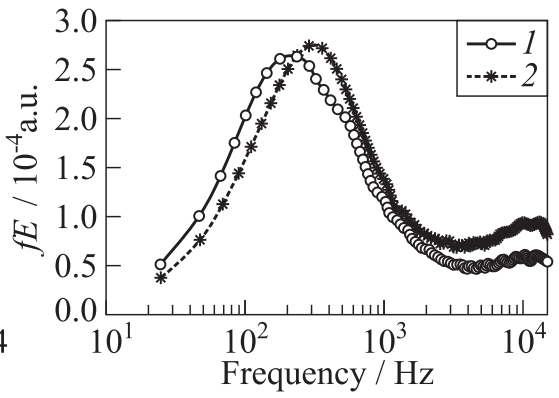

(b)

Figure 9 Shock position and frequency measurements in the symmetry plane of the wind tunnel (1 - AJVGs off and 2 - AJVGs on): (a) RMS of the HWA-signal for different positions with and without AJVG control; and (b) premultiplied HWA spectrum for the reflected shock with and without jets 
where $h$ is defined as the maximum height of the dividing streamline [3]. This can be demonstrated as follows. To determine the effect of the observed change in bubble height and shock frequency at constant reference velocity on the Strouhal number, one can write (using central differencing):

$$
\frac{2 \Delta \mathrm{St}}{\mathrm{St}_{0}-\mathrm{St}}=\frac{2 \Delta h}{h_{0}+h}+\frac{2 \Delta h}{f_{0}+f} .
$$

The maximum height of the dividing streamline was found to be, respectively, $h_{0}=7.4 \mathrm{~mm}$ for the reference case, and an average height of $h=5.0 \mathrm{~mm}$ for the case with jets $\left(h_{\max }=5.7 \mathrm{~mm}\right.$ for $L_{\max }$ and $h_{\min }=4.2 \mathrm{~mm}$ for $\left.L_{\min }\right)$. Referring to Fig. $9 b$, the frequency is, respectively, $f_{0}=200 \mathrm{~Hz}$ for the case without AJVGs and approximately $f=300 \mathrm{~Hz}$ with AJVGs. This leads to a negligible variation of the Strouhal number (approximately 1\%) compared to a significant change in height and frequency (both about 40\%). It is pointed out that the frequency measurements were made only in the symmetry plane of the wind tunnel. The hypothesis of a variation of the shock frequency in the spanwise direction could affect this result.

The present data do, however, confirm the qualitative trend that a higher frequency corresponds to a smaller interaction length and a smaller bubble size, at least in the case of a well developed mean separation bubble. Such a modification of the frequency should be taken into account for practical control applications.

\section{RESULTS OF RECAPITULATION}

\subsection{Effect of Jets on the Boundary Layer}

The horizontal and vertical plane PIV measurements indicate that the AJVGs induce the formation of large longitudinal vortices. These vortices entrain highvelocity fluid from higher up in the boundary layer and, hence, induce locally higher velocities closer to the wall. The effect of the jets is only noticeable up to $y / \delta=0.8$. Above this height, the boundary layer seems not or only slightly affected. In the 'wake' of the jets, the boundary layer profile fullness is significantly lower than the reference boundary layer. At the same time, the boundary layer profile in-between the jets is fuller than the reference boundary layer. This coincides with a decrease in friction coefficient and friction velocity for the jet 'wake' and a respective increase of both quantities for the fullest profile in-between the jet 'wakes.' The inverse effect is induced on the shape factor, which increases for the slim profile and decreases for the full profile. Moreover, the jets also induce a slight skewing of the flow in the upstream boundary layer, deflecting the flow sideways by approximately $2.8^{\circ}$ at 1 -millimeter height from the wall. 


\subsection{Effect on the Separation}

The effect of the jets is more pronounced on the separation bubble, the modifications of the incoming boundary layer leads to a global three-dimensionalization. The overall separation length is reduced since the mean separation line is moved downstream while the reattachment is moved upstream. The corrugation of the separation line is more significant than the corrugation of the reflected shock. The most upstream separation point with AJVGs corresponds to most upstream point for the reference interaction. The reattachment line is not corrugated and no trace of the jets is observed after reattachment. The reattachment location is independent of the separation location.

Beyond these global properties, the following conclusion can be made on the effect of the spanwise modulation induced by the jets:

- the fullest boundary layer profile corresponds to the smallest separation length and the most downstream reflected shock position;

- the slimmest profile (the jet wake) corresponds to the largest separation length and the most upstream reflected shock position; and

- the height of the separation bubble is also reduced, with the largest reduction corresponding to the smallest separation length.

The most important effect of the AJVGs seems to be due to the integral boundary layer parameters and the mean velocity profile. A reduction in separation length is directly linked to the shape factor and the friction coefficient.

\subsection{Effect of Jets on the Reflected Shock}

The jets cause an overall corrugation of the reflected shock over its full height. This effect is observed as a thickening of the shock in the Schlieren images, and an undulation of the shock foot in the horizontal plane PIV measurements. The interaction length (distance at the wall of the extrapolated shocks) is only mildly affected, and no increase in shock excursion amplitude has been observed. The HWA results show an increase in reflected shock frequency caused by the AJVGs, in combination with a downstream displacement of the shock.

This is in agreement with the properties of the Strouhal number for the shock frequency: a higher frequency corresponds to a smaller interaction length and a smaller bubble size, at least in the case of a well developed mean separation bubble. 


\section{CONCLUDING REMARKS}

The results indicate that AJVGs, which are inclined under $45^{\circ}$ with respect to the wall and blow in transverse direction, generate two longitudinal counterrotative vortices per air jet. The vortices are of unequal strength, with the stronger vortex located above jet and the weaker vortex between the jet and the wall. The rotation rate of these vortices is small, notwithstanding the large injection pressure and a significant modification of the upstream boundary layer structure. Consequently, only a limited amount of mixing should be expected. However, it has been shown that the angular displacement induced by the flow is sufficiently large to entrain high-speed flow towards to wall, hence increasing the fullness of the boundary layer profile. At the same time, the opposite effect is observed in the wake of the jets, where the fullness is decreased.

No significant effect has been observed of the AJVGs on the shock excursion amplitude and position. However, the AJVGs do reduce the separation bubble size without suppressing it. As a direct consequence of the reduction in bubble size, the shock frequency is increased by $50 \%$.

\section{ACKNOWLEDGMENTS}

This work was carried out with support from a grant of the European STREP UFAST (contract No. AST4-CT-2005-012226). Their support is gratefully acknowledged. These results were presented at the 3rd EUCASS Conference, 6-9 July, 2009, Versailles.

\section{REFERENCES}

1. Dolling, D. S. 2001. Fifty years of shock-wave/boundary-layer interaction research: What next. AIAA J. 39(8):1517-31.

2. Ganapathisubramani, B., N.T. Clemens, and D.S. Dolling. 2007. Effects of upstream coherent structures on low-frequency motion of shock-induced turbulent separation. 45th AIAA Aerospace Sciences Meeting and Exhibit. Reno, Nevada.

3. Piponniau, S., J. P. Dussauge, J. F. Debiève, and P. Dupont. 2009. A simple model for low-frequency unsteadiness in shock-induced separation. J. Fluid Mech. 629:87108.

4. Souverein, L. J., P. Dupont, J.F. Debiève, J. P. Dussauge, B. W. Van Oudheusden, and F. Scarano. 2009. Effect of interaction strength on shock wave boundary layer interaction: Unsteady behavior. AIAA Paper No. 2009-3715. 
5. Dupont, P., S. Piponniau, A. Sidorenko, and J. F. Debiève. 2008. Investigation of an oblique shock reflection with separation by PIV measurements. AIAA J. 46(6).

6. Elena, M. G. Tedeschi, and H. Gouin. 1999. Motion of tracer particles in supersonic flows. Exper. Fluids 26(4):288-96.

7. Dupont, P., C. Haddad, and J. F. Debiève. 2006. Space and time organization in a shock induced boundary layer. J. Fluid Mech. 559:255-77.

8. Dussauge, J. P., P. Dupont, and J.F. Debiève. 2006. Unsteadiness in shock wave boundary layer interactions with separation. Aerospace Sci. Technol. 10:85-91. 